\title{
Clinical and cost effectiveness of arthritis gloves in rheumatoid arthritis (A-GLOVES): randomised controlled trial with economic analysis
}

Alison Hammond ${ }^{1 *}$ (D, Yeliz Prior ${ }^{1}$, Sarah Cotterill ${ }^{2}$, Chris Sutton ${ }^{2}$, Elizabeth Camacho ${ }^{3}$, Calvin Heal ${ }^{2}$, Jo Adams ${ }^{4}$, Yvonne Hough ${ }^{5}$, Terence W. O'Neill ${ }^{6,7}$ and Jill Firth ${ }^{8}$

\begin{abstract}
Background: Arthritis (or compression) gloves are widely prescribed to people with rheumatoid arthritis and other forms of hand arthritis. They are prescribed for daytime wear to reduce hand pain and improve hand function, and/ or night-time wear to reduce pain, improve sleep and reduce morning stiffness. However, evidence for their effectiveness is limited. The aims of this study were to investigate the clinical and cost effectiveness of arthritis gloves compared to placebo gloves on hand pain, stiffness and function in people with rheumatoid arthritis and persistent hand pain.

Methods: A parallel randomised controlled trial, in adults ( $\geq 18$ years) with rheumatoid or undifferentiated inflammatory arthritis at 16 National Health Service sites in the UK. Patients with persistent hand pain affecting function and/or sleep were eligible. Randomisation (1:1) was stratified by recent change (or not) in medication, using permuted blocks of random sizes. Three-quarter-finger length arthritis gloves (Isotoner ${ }^{\oplus}$ : applying $23-32 \mathrm{mmHg}$ pressure) (intervention) were compared to loose-fitting placebo gloves (Jobskin ${ }^{\oplus}$ classic: providing no/minimal pressure) (control). Both gloves (considered to have similar thermal qualities) were provided by occupational therapists. Patients and outcome assessors were blinded; clinicians were not. The primary outcome was dominant hand pain on activity (0-10) at 12 weeks, analysed using linear regression and intention to treat principles.
\end{abstract}

Results: Two hundred six participants were randomly assigned (103 per arm) and 163 (84 intervention: 79 control) completed 12-week follow-up. Hand pain improved by 1.0 (intervention) and 1.2 (control), an adjusted mean difference of 0.10 ( $95 \%$ Cl: 0.47 to $0.67 ; p=0.72$ ). Adverse events were reported by $51 \%$ of intervention and $36 \%$ of control group participants; with 6 and $7 \%$ respectively, discontinuing glove wear. Provision of arthritis gloves cost $£ 129$, with no additional benefit.

Conclusion: The trial provides evidence of no clinically important effect of arthritis gloves on any of the trial outcomes (hand pain, function and stiffness) and arthritis gloves are not cost-effective. The clinical and cost-effectiveness results support ceasing provision of arthritis gloves in routine clinical practice. Funding: National Institute for Health Research.

Trial registration: ISRCTN, ISRCTN25892131; Registered 05/09/2016: retrospectively registered.

Keywords: Rheumatoid arthritis, Rehabilitation, Hand, Pain, Orthotic devices, Clinical trial

\footnotetext{
* Correspondence: A.hammond@salford.ac.uk

${ }^{1}$ Centre for Health Sciences Research, School of Health and Society,

University of Salford, Allerton Building, Frederick Road, Salford M6 6PU, UK

Full list of author information is available at the end of the article
}

(c) The Author(s). 2021 Open Access This article is licensed under a Creative Commons Attribution 4.0 International License, which permits use, sharing, adaptation, distribution and reproduction in any medium or format, as long as you give appropriate credit to the original author(s) and the source, provide a link to the Creative Commons licence, and indicate if changes were made. The images or other third party material in this article are included in the article's Creative Commons licence, unless indicated otherwise in a credit line to the material. If material is not included in the article's Creative Commons licence and your intended use is not permitted by statutory regulation or exceeds the permitted use, you will need to obtain permission directly from the copyright holder. To view a copy of this licence, visit http://creativecommons.org/licenses/by/4.0/ The Creative Commons Public Domain Dedication waiver (http://creativecommons.org/publicdomain/zero/1.0/) applies to the data made available in this article, unless otherwise stated in a credit line to the data. 


\section{Introduction}

Rheumatoid arthritis (RA) affects $1 \%$ of the world's population [1]. Disease modifying anti-rheumatic drugs (DMARDs) are prescribed as soon as possible to control symptoms, including to those with persistent synovitis where other pathologies are ruled out but not yet meeting criteria for RA, i.e. undifferentiated inflammatory arthritis (UIA). Functional ability can still deteriorate even though disease activity is controlled [2]. Over $90 \%$ of people with RA and UIA report hand symptoms of pain, stiffness, muscle weakness, paraesthesia, and difficulty making a fist [3]. Most have bilateral hand symptoms, resulting in difficulties with work, everyday activities and leisure.

Arthritis gloves (or compression gloves) have been commonly prescribed since the 1980's for people with RA and UIA in the United Kingdom (UK), North America and Europe [4-7]. A survey of occupational therapists in the UK identified that the most common model and make prescribed are three-quarter length finger Isotoner ${ }^{\oplus}$ gloves (Fig. 1) [4]. They are prescribed for daytime wear to reduce hand pain and improve hand function, and/or night-time wear to reduce pain, improve sleep and reduce morning stiffness $[4,5]$. The second most common are three-quarter length finger oedema gloves, of which there are several makes available [4]. The mechanism whereby arthritis gloves are thought to impact on hand symptoms is through compression, which: removes extracellular fluid, thus reducing pain, stiffness and improving finger movement; and increases blood flow, thus increasing warmth and reducing pain $[6,7]$. In clinical practice, therapists ensure a "snug fit" around the proximal interphalangeal joints (PIPJs), metacarpophalangeal joints (MCPJs) and across the dorsum of the hand, i.e. to ensure enough compression is applied without restricting circulation to the fingers or causing pins and needles. It is unclear what a

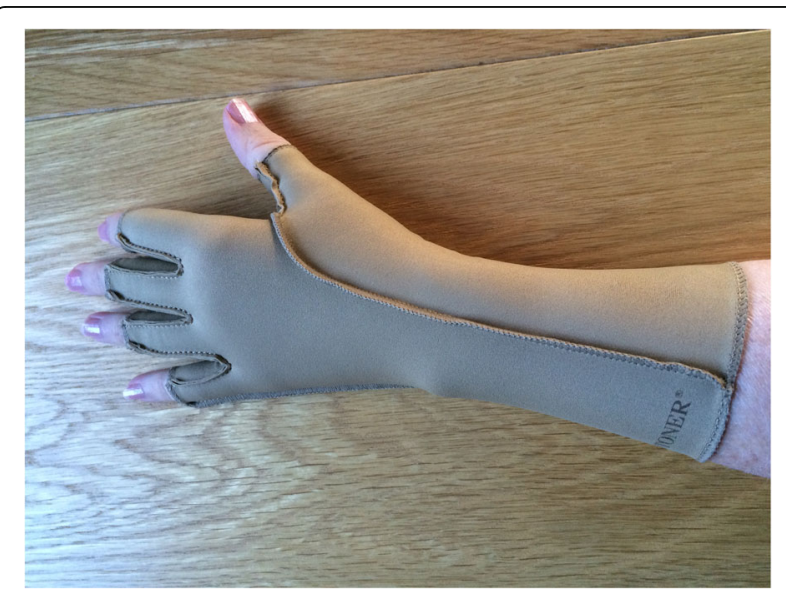

Fig. 1 Intervention $\left(\right.$ Isotoner ${ }^{\oplus}$ ) Arthritis Glove therapeutic level of compression is, as no physiological studies have been conducted. Manufacturers of commonly prescribed glove makes report pressures of 15 to $32 \mathrm{mmHg}[8,9]$. The amount of compression applied depends on: the amount of elastane in the glove fabric; the individual's hand size and shape; and the glove fit. Isotoner ${ }^{\bullet}$ gloves have the highest amount of elastane content of those currently on the market. According to the manufacturers' specifications, Isotoner ${ }^{ø}$ gloves exert the highest pressure [8, 9].

Arthritis gloves are considered a comparatively lowcost, quickly provided treatment for pain relief, although they are a recurrent cost as they need replacing every six-months. Gloves are also commonly prescribed to people with other forms of inflammatory arthritis and with hand osteoarthritis. The global market for arthritis gloves is rising [10] and millions of people with hand arthritis worldwide purchase these gloves for themselves.

Systematic review evidence is inconclusive about the effectiveness of arthritis gloves [5]. Previous trials were small ( $n=8$ to 24 ), evaluated a variety of full-length finger arthritis gloves in RA for night-time use only [6, $7,11-13$ ], and had moderate to high risk of bias [5]. Three trials, with moderate risk of bias, showed significant reductions in PIPJ circumference. However, this was only 0.7 to $1.15 \mathrm{~mm}$ and it is questionable what the benefits of this were [5]. Trials were inconclusive about effects on nocturnal hand pain, morning stiffness and hand swelling. No benefits were identified in range of motion, dexterity, grip and pinch strength [5]. One trial found arthritis gloves and thermal gloves had similar effects in reducing nocturnal hand pain and hand stiffness [6]. Gloves' effect on day-time hand pain, the most common reason for prescription nowadays, has not been evaluated.

We conducted a pre-test post-test feasibility trial to evaluate Isotoner ${ }^{\bullet}$ arthritis gloves, over a four-week period, in people with RA and UIA in 10 Rheumatology occupational therapy departments. In this we: developed a treatment manual, based on therapists' expertise in providing arthritis gloves, to standardise delivery; developed an arthritis glove information sheet for participants; developed and tested trial procedures; identified the most relevant outcome measure considered by participants was day-time hand pain; and that this and other secondary outcomes deemed most relevant were self-report and therefore collectable using reliable, valid patient reported outcome measures. Participants reported they liked the warmth, comfort and gentle support provided by the arthritis gloves [14]. The results of this trial indicated that the procedures were feasible and there were some improvements in hand pain, stiffness and function, with the caveat that there was no control group. This supported the need for a definitive trial. 
We sought the input of our clinical stakeholders, participating therapists and our patient and public involvement (PPI) representatives throughout this research. We conducted a series of focus groups to discuss the feasibility study results and design the A-Gloves trial, to ensure it would be acceptable to both patients and participating clinicians. We discussed what the comparator intervention should be: usual care; ordinary gloves plus usual care; or placebo gloves plus usual care. Our patient research partners emphasised the importance of comparing arthritis gloves with placebo gloves, and that these should be credible (i.e. another model of arthritis glove which was loosely fitted to apply no pressure but provide similar warmth) to ensure that the effects of wearing a "medical device" were controlled for. We discussed the length of follow-up required. The PPI group and therapists emphasised glove wear should increase gradually over the first few weeks, in order to become accustomed to glove effects. Therapists reported patients usually start to report any benefits, or adverse effects, by a four-week review appointment. As gloves are intended to be worn long-term, we selected a 12-week follow-up as: allowing several weeks for glove tolerance to develop; participants to experience effects of regular wear for up to 2 months across a range of activities; and being a feasible time-scale within the funding period. Therapists highlighted the importance of ensuring participating therapists understand that there is only low-level evidence about arthritis gloves currently, it is unclear if arthritis gloves are effective and therefore there is clinical equipoise between arthritis and placebo gloves (i.e. a placebo glove providing warmth is an ethically acceptable alternative). This was considered essential as the participating therapists all normally regularly provided arthritis gloves as part of usual care. Accordingly, we needed to facilitate therapists to overcome personal preferences to provide arthritis gloves, be ethically willing to enrol participants and provide placebo gloves in a trial, be able to provide placebo gloves in a credible way and not have prior expectations of the trial findings.

In the Arthritis Gloves (A-GLOVES) trial we aimed to investigate, for people with RA and UIA with persistent hand pain, the comparative clinical and costeffectiveness of providing arthritis gloves or placebo gloves in addition to usual care.

\section{Methods}

\section{Study design and ethics}

The A-GLOVES trial was a pragmatic, multicentre, investigator-blind, parallel-group randomised controlled trial. The trial was conducted in rheumatology occupational therapy departments in 16 National Health Service sites in England and Scotland. The trial was approved by the North of Scotland National Research
Ethics Service Committee (REC reference 15/NS/0077). The full protocol has been published [15].

\section{Participants}

Patients were eligible for inclusion if aged 18 years or older, diagnosed with RA or UIA by a Rheumatology consultant, with persistent pain in the PIPJs or MCPJs causing at least one of: difficulty using the hands during the day (for day wear); disturbed sleep (for night wear); and limited ability to use the hands in the morning (for night wear). We excluded patients diagnosed with other inflammatory forms of arthritis affecting the hands (e.g. psoriatic arthritis, gout, ankylosing spondylitis); severe Raynaud's disease, hand circulatory disturbances, hand neuropathies or hand deformities; and any contraindications to glove-wear (e.g. eczema, broken skin). Participants should not previously have worn arthritis gloves.

We asked clinicians to identify potentially eligible patients during clinic visits or from therapy records. Patients were given an invitation letter and information sheet. If interested they saw a research practitioner or occupational therapist to discuss the trial, check eligibility, complete consent and study registration and receive the baseline questionnaire (completed at home then mailed to the research co-ordinating centre). Participants at screening who had recently received a steroid injection or started oral steroids were deferred for 6 weeks after injection or drug start, and re-screened to check for eligibility. Steroids could be a confounding variable as they quickly improve hand symptoms [16].

\section{Randomisation and blinding}

Participants were randomly assigned (1:1) to the intervention or control group, stratified by whether the participant had a change in or new medication (DMARDs or biologics) or not within the last 3 months, using permuted blocks of random sizes. Randomisation was completed by the Lancashire Clinical Trials Unit using Sealed Envelope, a web-based central randomisation service [17]. Participants were accumulated into both groups from start of recruitment at each site. After randomisation, allocation was unblinded to therapists delivering treatment. Participants were not blinded to group allocation. The study was described as comparing the effects of two types of arthritis glove without divulging the differences. Therapists were asked not to use the term "compression gloves" to participants, in order to reduce the risk of unblinding. Investigators and data management staff were blinded to group allocation. Data were analysed blinded to group allocation.

\section{Interventions}

Following randomisation, a referral was sent to the treating therapist, including group allocation. Gloves were 
fitted within 3 weeks, with a review appointment 2 to 4 weeks later to check for glove fit and any adverse events. We tested the intervention gloves against placebo gloves, in order to control for therapist time, attention and effects of receiving a medical device.

Participants in the intervention group, received correctly fitted three-quarter length finger Isotoner ${ }^{\circ}$ gloves. These are made of $80 \%$ nylon and $20 \%$ elastane. These were selected for testing as they exert the highest level of pressure of arthritis gloves available, at $23-32 \mathrm{mmHg}$ [8]. If compression is the mechanism of action of arthritis gloves, then these gloves are more likely to lead to effects being detected. Additionally, as these are the most popular glove prescribed, testing Isotoner ${ }^{\bullet}$ gloves reflects UK clinical practice [4]. The size range comfortably fits hands up to $23.5 \mathrm{~cm}$. MCPJs circumference. Clinically, fitting Isotoner gloves is not possible for people with larger hands, as they are too tight and can cause problems with pins and needles, numbness or limit finger circulation (Fig. 1).

Participants in the control group received loose-fitting three-quarter length finger Jobskin ${ }^{\circ}$ classic oedema gloves, made of $89 \%$ nylon and $11 \%$ elastane (placebo gloves). When fitted correctly, these exert $15-25 \mathrm{mmHg}$ pressure [9]. However, these were fitted at least one size too large and exerted no pressure. A focus group of RA patients and expert rheumatology occupational therapists chose the Jobskin ${ }^{\circ}$ gloves, worn loosely, as a credible placebo glove because of sufficiently similar appearance, material and warmth to the intervention gloves. Additionally, Jobskin ${ }^{\bullet}$ gloves have the largest size range available of arthritis glove makes prescribed in clinical practice, accommodating hands up to $25.4 \mathrm{~cm}$. MCPJs circumference (Fig. 2).

At the first appointment, therapists measured the participant's MCPJs circumference to determine correct glove size, visually checked appropriate fit and discussed hand symptoms and function to determine an individual wear regimen. Participants in either group, with hands larger than $23.5 \mathrm{~cm} \mathrm{MCPJs} \mathrm{circumference,} \mathrm{were} \mathrm{not} \mathrm{fit-}$ ted with gloves as: the Isotoner ${ }^{\circ}$ intervention gloves would be too tight; and the largest size of placebo glove insufficiently loose. Previous studies have reported difficulties fitting gloves for those with large hands, with some men reporting gloves being too short or small [5]. Most participants were issued with gloves for both hands for day and night wear. However, some were provided with only one glove (usually the dominant hand); or advised to wear gloves during the day or night only, i.e. consistent with routine clinical practice. Participants could wear gloves for most of the day and all night but informed not to wear $24 \mathrm{~h}$ a day. Participants were given booklets about hand self-management, including joint protection [18], and hand exercise [19] and asked to do

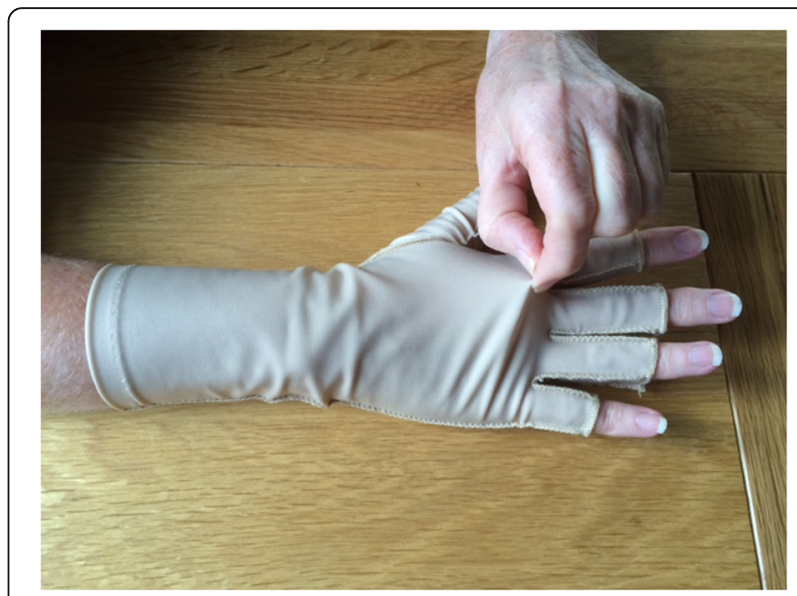

Fig. 2 Placebo Glove (loose-fitting Jobskin ${ }^{\oplus}$ classic oedema glove: control group)

hand exercises daily. We have made available the Arthritis Gloves Occupational Therapist Provision Manual describing the clinical protocol [20].

Participants continued to receive their Rheumatology and Occupational Therapy department's usual care. The therapist could provide advice and training for up to $1 \mathrm{~h}$ in joint protection and hand exercises when providing gloves, (if needed, if the participant had not already received these), reflecting clinical practice [4, 14]. Other therapies could be provided.

\section{Therapist training}

Prior to the trial starting, we explained to therapists: the existing research about arthritis gloves, the lack of good quality and contradictory evidence for efficacy and effectiveness (clinical equipoise); the trial design to ensure that they understood the reasons for testing arthritis against placebo gloves and that warmth might be a contributory mechanism, meaning placebo gloves are an acceptable comparator; and discussed any concerns about providing placebo gloves. Therapists agreed to take part following consideration of the trial design. Prior to attending the trial training course, therapists were asked to read two articles: a systematic review of arthritis gloves [5] and an article exploring therapists' views about placebo splint provision within a trial [21]. They then attended a one-day training course including: education about randomised controlled trials; the A-Gloves trial procedures, theoretical and practical training in intervention and placebo glove fitting, in order to standardise the interventions; and a focus group about their views of providing placebo gloves and personal, service and research methodology issues in relation to their trial participation [22]. Training explained the placebo effect and emphasised: the importance of ensuring placebo gloves were a loose fit so that no pressure was being 
applied, to avoid treatment contamination in the control group; and how to answer control participants' queries related to the loose-fit of the placebo gloves, in order to reduce the risk of control participant stopping glovewear. The accompanying glove provision manual detailed procedures, glove-fitting and issues of providing placebo gloves [20].

\section{Data collection}

Baseline data were collected using a postal selfcompleted questionnaire. Randomisation occurred immediately after receipt. Follow-up data were obtained 12 -weeks post-randomisation by postal self-completed questionnaire. After 1 week, participants were reminded by e-mail, text or telephone to return the questionnaire. If no response was received: after 2 weeks, participants were sent a second copy of the questionnaire; and after 4 weeks they were contacted by telephone to obtain a minimal data set, if possible.

\section{Outcomes}

The primary outcome measure, at 12-weeks postrandomisation, was hand pain in the dominant hand during the daytime when doing moderate hand activities (e.g. housework, cooking, DIY, gardening), measured on a numeric rating scale (NRS), with anchor points of no pain (0) and severe pain (10). Minimal clinically important differences (MCID) for pain scales in RA are 1.1 points on a $0-10$ NRS $[23,24]$. Hand pain was selected because it is the most common reason therapists provide gloves and the symptom that patients most frequently describe as benefiting from glove use $[5,6,14]$.

Secondary outcomes were: non-dominant hand pain in the day (0-10 NRS), dominant and non-dominant hand pain at night (0-10 NRS); hand stiffness (0-10 NRS), owing to a lack of predefined MCID this was considered as $\geq 0.5 \mathrm{SD}$ of mean baseline score $[25,26]$, i.e. in this trial $=1.4$ points); duration of hand early morning stiffness (minutes); hand function (Measure of Activity Performance-Hand, with higher scores denoting worse hand function and Minimal Detectable Change (at 95\% confidence interval: $\mathrm{MDC}_{95}$ ) of 3.99 [27]; and Michigan Hand Outcomes Questionnaire, with higher scores denoting better hand performance (apart from the hand pain sub-scale), with an MCID of 13 points [28, 29]; and disability (Health Assessment Questionnaire, with higher scores denoting worse function [30]. Participants completed the EQ-5D-3L [31] as a measure of health benefit for the economic evaluation and reported their use of health and social care services at baseline and follow-up. Additionally, at 12-week follow-up, we asked participants about: glove use; perceptions of glove-wear; and new steroid prescription (injection or oral). Therapists recorded glove provision, wear regimen and occupational therapy provided as part of usual care on standardised treatment logs. These were reviewed to identify documentary evidence of glove provision, treatment duration, and any adverse events. A detailed description of outcome measures was published in the trial protocol [15]. Those questionnaire items developed specifically for the trial were constructed with the assistance of the patient research partners (see study questionnaire in Supplementary Materials).

\section{Sample size}

Using data from a pilot study [14], the mean change in hand pain NRS (measured 4 weeks post-intervention) was -1.03 (SD 2.22). The $80 \%$ upper one-sided confidence limit of the estimated SD, i.e. 2.48 was used. To identify a MCID of 1.1-point, $\mathrm{SD}=2.48$, significance level of 0.05 and $80 \%$ power, 80 participants per group were required. Allowing for $22 \%$ missing 12 -week primary outcome data, the target sample size was 205 randomised participants.

\section{Statistical and economic analysis}

The analysis followed a pre-specified statistical analysis plan and was by intention to treat, without imputation of missing data. STATA V.14 was used [32]. Baseline characteristics were described, overall and by group, reporting mean (standard deviations), median (IQR) or number (proportion), as appropriate. Primary effectiveness analysis used linear regression to estimate an adjusted mean difference comparing dominant hand pain during activity at 12 weeks (primary outcome) between groups, adjusting for baseline scores and the stratification variable (recent DMARD changes or not). Secondary analyses used appropriate modelling approaches (multiple linear regression, logistic regression or ordinal logistic regression), to estimate the effect of group allocation on the other health outcomes at 12 weeks. A sensitivity (per protocol) analysis omitted those who did not receive gloves, or who self- reported being prescribed steroids (oral or injection) during the trial. Data were analysed by person and using dominant hand results, as reported by the participant.

The economic analysis compared the costs and health benefits of the gloves from a health and social care perspective. The primary economic analysis was based on 151 complete cases (i.e. participants with no missing data). Sensitivity analysis explored the cost effectiveness of arthritis gloves using the primary outcome measure, in the sub-group of participants who were treated as per the trial protocol (i.e. received gloves), and under alternative models of glove provision.

Intervention costs were estimated from individual patient treatment logs, for the number of gloves provided, assuming one visit to an occupational therapist. 
Published unit costs were used to calculate total costs for the intervention and healthcare utilisation in both groups [33, 34]. Quality-adjusted life-years (QALYs) were estimated from the EQ-5D-3L data, using an area under the curve approach and published utility tariffs for England [35]. Linear regression analysis was used to estimate net QALYs and a generalised linear model with log link and gamma family was used to estimate net costs for the arthritis gloves compared to the placebo gloves, adjusted for baseline values and stratification variable. Bootstrapping ( $n=10,000$ simulations) was used to estimate the probability that arthritis gloves were cost-effective at different willingness to pay thresholds (WTPTs).

\section{Results}

\section{Recruitment}

Figure 3 shows the patient flow through the trial. Between February 2016 and May 2017, 206 participants were recruited and randomised, with 103 in the intervention and 103 in the control groups. Within the intervention group, 102 (99\%) received Isotoner gloves. Within the control group, 88 (85\%) received the placebo gloves: 10 could not be fitted with gloves due to larger hand size. At 12-week follow-up, data were received from $84(82 \%)$ in the intervention and $79(77 \%)$ in the control group. Those not fitted with gloves continued to be followed up (Supplementary Table S1).

\section{Participants}

The median age of participants was 59 years, over $80 \%$ were women and a third employed. Most were diagnosed with RA (86\% control; 84\% intervention). Median time since diagnosis was 4 years and most $(90 \%)$ were prescribed DMARDs or biologic drugs (Table 1).

\section{Glove provision and other therapy}

Most participants (78\%) received gloves within 3 weeks of referral. All received gloves for their dominant hand, and most in both groups for both hands (95/102 (93\%) intervention; $n=83 / 88$ (94\%) control). In both groups, $73 \%$ were recommended to wear gloves during the day. Two-thirds of those responding at 12-weeks answered the adherence items. For the remainder, glove wear was unknown.

Self-reported glove wear was very similar between groups for both hands at on average: $5 \mathrm{~h}$ during the daytime on 5 days/ week; and $6 \mathrm{~h}$ at night for 5 nights/week (Table 2). On average, the intervention group wore arthritis gloves somewhat less often at night, compared to placebo glove wear in the control group. At 12-week follow-up, there were no differences between groups in treatment duration or receiving steroids (Supplementary Table S2).

\section{Primary outcome}

At baseline both groups had moderate day-time levels of dominant hand pain during activity (6.3 on the $0-10$ NRS). At 12-week follow-up ( $n=154)$, day-time dominant hand pain reduced in both groups, with the intervention group $(n=84)$ reducing slightly less than the control group ( $n=79)$ (1.0 versus 1.2 points). There was no evidence of a difference between the intervention and control gloves, after adjusting for the stratification variable and baseline pain scores. The adjusted difference of 0.1 (favouring the control group) between groups (95\% CI -0.47 to 0.67 ) was not statistically significant nor clinically relevant (as the largest plausible positive effect of the intervention gloves was 0.47 units, less than half the MCID of 1.1 points) (Table 3). In the sensitivity (per protocol) analysis (i.e. only those receiving the correct gloves and who did not receive steroids), there was also no statistically significant difference between groups $(0.2$ points, CI -0.41 to $0.81 ; p=0.51)$. No further analysis adjusting for effects of glove-wear was conducted. As there were no substantive differences in self-reported frequency of dominant hand glove wear between groups, this would have minimal impact on the estimated between group differences in the primary outcome.

\section{Secondary outcomes}

Both groups reported similar small levels of improvement in hand symptoms of non-dominant hand pain on activity, dominant and non-dominant hand pain at night, hand stiffness, duration of morning hand stiffness, and self-reported hand status, with no statistically significant or clinically important differences between groups. Both groups reported similar small improvements in hand function and disability and with no statistically significant or clinically relevant differences between groups (Table 3).

In both groups, in those reporting perceived effects, over $70 \%$ considered wearing gloves gave warmth and comfort, were beneficial, and they would continue to wear them (Table 2), supporting the qualitative study [36]. Forty-one per cent in both groups thought they helped them sleep better. Perceptions of glove-wear will be reported in detail elsewhere.

\section{Adverse events}

In the intervention group, 47 (51\%) reported an adverse event compared to 29 (36\%) in the control group, with the most common in both groups being sleep disturbance as the gloves made hands feel hot and itchy. The intervention group reported more adverse events of pins and needles, numbness or fingertip discolouration (26 events) than the control group (7 events) (Supplementary Table S3). Similar numbers discontinued glove-wear 


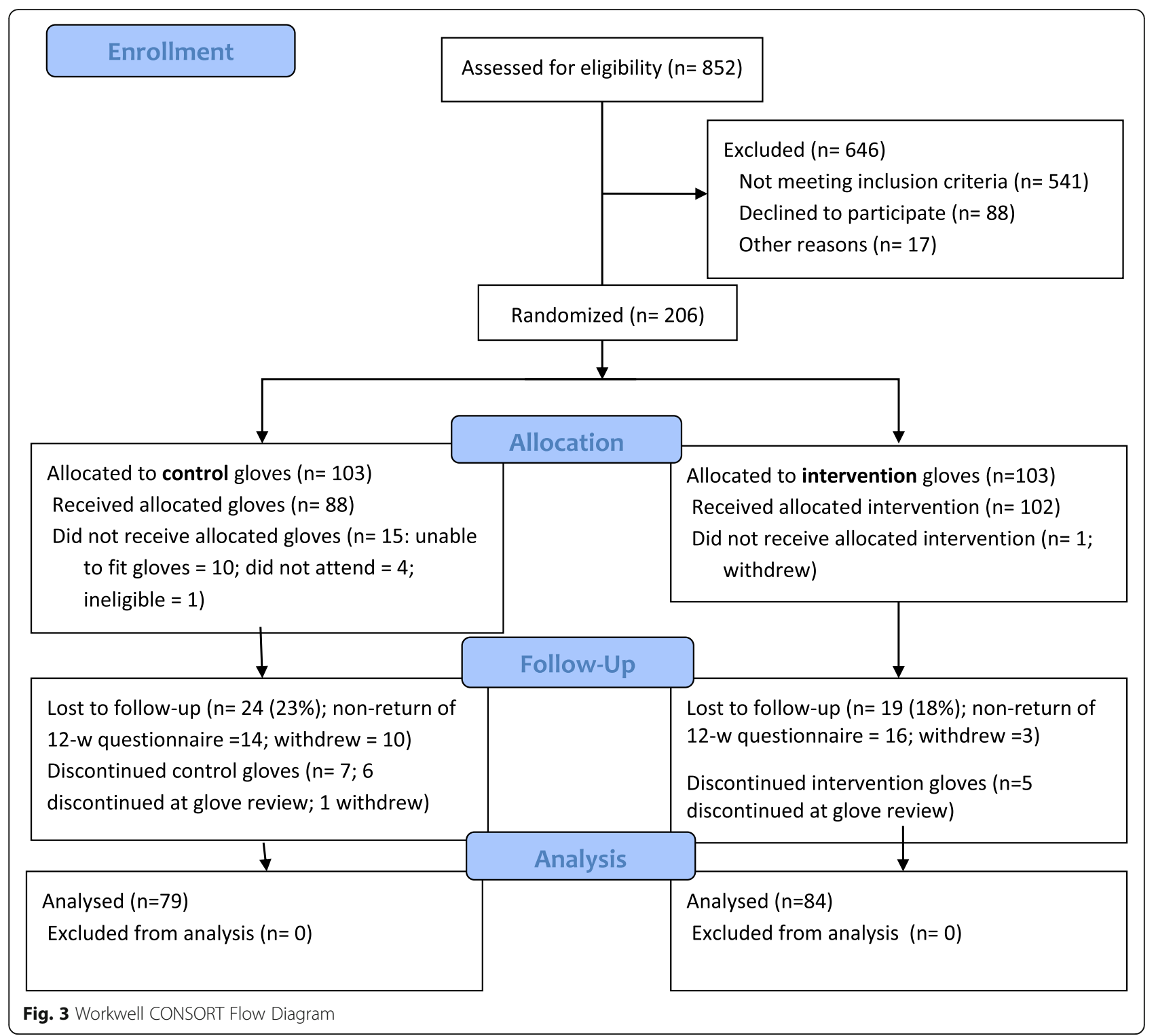

at the glove review appointment due to adverse events ( $n=7(7 \%)$ control; $n=6$ (6\%) intervention, Fig. 3).

\section{Cost-effectiveness}

Both control and intervention groups had the same health utility at baseline and accrued the same number of QALYs during follow-up (Table 4).

Use of physiotherapy services was higher in the intervention group (Supplementary Table 4). Costs associated with healthcare services used are shown in Table 4. The cost of providing placebo gloves is not included as these would not normally be provided in the NHS. The main difference in costs between groups was that of providing the intervention gloves. The results of the incremental cost-effectiveness analysis are reported in Table 5 . The intervention gloves are associated with higher costs but comparable benefits to the placebo gloves and therefore unlikely to be cost-effective. The gloves had an incremental cost-effectiveness ratio (ICER) of $£ 83,700$ per QALY gained. The intervention gloves have a probability of $0.19-0.29$ of being cost-effective if decision-makers are willing to pay $£ 20-30,000 / Q A L Y$, respectively. Sensitivity analyses confirmed that the intervention gloves are not likely to be cost-effective (results reported in full in Supplementary Table S5).

\section{Discussion}

These results showed that, for people with RA or UIA, with moderate to severe hand pain, correctly fitted arthritis gloves led to slight improvements in hand pain (day or night), stiffness and function. However, wearing loose-fitting placebo gloves led to similar slight benefits. 
Table 1 Baseline participant characteristics of the intention-to-treat population $(n=206)$

\begin{tabular}{|c|c|c|}
\hline & Control $(n=103)$ & Intervention $(n=103)$ \\
\hline Age (years): median (IQR) & $60(51,68)$ & $58(51,67)$ \\
\hline Sex: female (\%) & $82(80 \%)$ & $84(82 \%)$ \\
\hline \multicolumn{3}{|l|}{ Diagnosis: } \\
\hline RA & $89(86 \%)$ & 87 (84\%) \\
\hline UIA & $14(14 \%)$ & $16(16 \%)$ \\
\hline \multicolumn{3}{|l|}{ Hand dominance: } \\
\hline Right (\%) & 92 (89\%) & 93 (90\%) \\
\hline Left (\%) & $9(9 \%)$ & $3(3 \%)$ \\
\hline Both (\%) & $2(2 \%)$ & $7(7 \%)$ \\
\hline Living status: Alone (\%) & $18(17 \%)$ & 19 (18\%) \\
\hline Living with children: yes (\%) & $10(10 \%)$ & $14(14 \%)$ \\
\hline \multicolumn{3}{|l|}{ Employment status: } \\
\hline Retired & $50(49 \%)$ & $44(43 \%)$ \\
\hline Due to ill health & $9(9 \%)$ & $18(17 \%)$ \\
\hline Other & $41(40 \%)$ & $26(25 \%)$ \\
\hline Full-time work & 24 (24\%) & $21(20 \%)$ \\
\hline Part-time work & $13(13 \%)$ & $18(17 \%)$ \\
\hline Homemaker & $9(9 \%)$ & $6(6 \%)$ \\
\hline Long-term sick & $2(2 \%)$ & $7(7 \%)$ \\
\hline Unemployed & $3(3 \%)$ & $3(3 \%)$ \\
\hline Self-employed & $1(1 \%)$ & $3(3 \%)$ \\
\hline Student & $0(0 \%)$ & $1(1 \%)$ \\
\hline Missing & 1. (1\%) & \\
\hline \multicolumn{3}{|l|}{ Symptom duration (months): } \\
\hline Median (IQR) & $60(24,168)$ & $72(18,174)$ \\
\hline \multicolumn{3}{|l|}{ Diagnosis duration (months): } \\
\hline Median (IQR) & $48(10,144)$ & $51(8,168)$ \\
\hline \multicolumn{3}{|l|}{ Medication regimen: } \\
\hline 0 DMARDs $^{a}$ & 10 & 11 \\
\hline 1 DMARD & 51 & 56 \\
\hline 2 or more DMARDs & 42 & 36 \\
\hline Biologics ${ }^{b}$ & 15 & 18 \\
\hline Started new/ changed DMARD or biologic in previous 3 months ${ }^{c}$ : yes (\%) & $34(33 \%)$ & $34(33 \%)$ \\
\hline
\end{tabular}

${ }^{\mathrm{a} D M A R D}$ Disease Modifying Anti-Rheumatic Drug; ${ }^{\mathrm{b}}$ participants were also prescribed a DMARD; ${ }^{\mathrm{c}}$ stratification variable

Improvements from both types of gloves were at, or only marginally above, the MCIDs for hand pain and hand function, and below for hand stiffness, with no significant or clinical differences between glove types. A high number experienced adverse events: a half of those wearing intervention and a third wearing control gloves. Both gloves led to similar levels of reporting about disrupted sleep. The intervention group experienced more neurological and circulatory adverse events resulting from the higher pressure applied by Isotoner ${ }^{\circ}$ gloves. Self-reported glove wear was similar between groups, apart from the Isotoner gloves being worn somewhat less at night by the intervention group. These neurological and circulatory adverse events are more likely to occur during prolonged periods of wear, such as at night. There was also some more reporting of hands becoming hot and itchy at night (especially in hot weather) in the intervention group. Both effects may have contributed to Isotoner ${ }^{\circ}$ gloves being worn less often at night in the intervention group. The arthritis gloves were not cost-effective. Our results indicate arthritis gloves should not be provided in routine clinical practice. This would 
Table 2 Recommended glove-wear regimen, self-reported glove wear and glove benefit (12- week follow-up: $n=154$ )

\begin{tabular}{|c|c|c|c|}
\hline & Control $\left(n=70^{\mathrm{a}}\right)$ : & Intervention $(n=84)$ : & $p$ \\
\hline \multicolumn{4}{|l|}{ Recommended glove-wear regimen: } \\
\hline - Day time only & $9(13 \%)$ & $20(24 \%)$ & 0.08 \\
\hline - Day and night & $42(60 \%)$ & $41(49 \%)$ & 0.17 \\
\hline - Night-time only & $19(27 \%)$ & $23(27 \%)$ & 0.97 \\
\hline \multicolumn{4}{|c|}{ Number of days gloves worn (day-time) per week in last 4 weeks: mean (SD) } \\
\hline - Dominant hand & $\begin{array}{l}5.2(1.8) \\
(n=47)\end{array}$ & $\begin{array}{l}5.3(1.8) \\
(n=56)\end{array}$ & 0.80 \\
\hline - Non-dominant hand & $\begin{array}{l}5.2(1.9) \\
(n=45)\end{array}$ & $\begin{array}{l}5.2(1.8) \\
(n=53)\end{array}$ & 0.94 \\
\hline \multicolumn{4}{|c|}{ Number of minutes gloves (worn (day-time) per day in last 4 weeks: Mean (SD) } \\
\hline - Dominant hand & $\begin{array}{l}310.0(218.9) \\
(n=48)\end{array}$ & $\begin{array}{l}316.9(215.1) \\
(n=57)\end{array}$ & 0.87 \\
\hline - Non-dominant hand & $\begin{array}{l}317.6(225.5) \\
(n=46)\end{array}$ & $\begin{array}{l}325.1(226.5) \\
(n=54)\end{array}$ & 0.87 \\
\hline \multicolumn{4}{|c|}{ Number of nights gloves worn per week in last 4 weeks: mean (SD) } \\
\hline - Dominant hand & $\begin{array}{l}5.5(1.9) \\
(n=52)\end{array}$ & $\begin{array}{l}4.9(2.1) \\
(n=55)\end{array}$ & 0.14 \\
\hline - Non-dominant hand & $\begin{array}{l}6.0(1.5) \\
(n=48)\end{array}$ & $\begin{array}{l}5.1(2.1) \\
(n=56)\end{array}$ & 0.01 \\
\hline \multicolumn{4}{|c|}{ Number of minutes gloves worn per night in last 4 weeks: Mean (SD) } \\
\hline - Dominant hand & $\begin{array}{l}412.6(131.7) \\
n=53\end{array}$ & $\begin{array}{l}384.4(136.5) \\
n=58\end{array}$ & 0.27 \\
\hline - Non-dominant hand & $\begin{array}{l}419.4(140.0) \\
n=50\end{array}$ & $\begin{array}{l}395.8(140.1) \\
n=58\end{array}$ & 0.38 \\
\hline \multicolumn{4}{|l|}{ Self-reported glove perceptions: } \\
\hline - Gloves are beneficial & $51(73 \%)$ & $61(73 \%)$ & 0.97 \\
\hline \multirow[t]{2}{*}{ - Will continue to wear gloves } & $50(72 \%)$ & $59(72 \%)$ & 0.94 \\
\hline & $(n=51)$ & $(n=61)$ & \\
\hline - Gloves give warmth & $41(80 \%)$ & $45(74 \%)$ & 0.41 \\
\hline - Gloves give comfort & $38(75 \%)$ & $52(85 \%)$ & 0.15 \\
\hline - Sleep better & $21(41 \%)$ & $25(41 \%)$ & 0.98 \\
\hline
\end{tabular}

${ }^{\mathrm{a}} 10$ control participants could not be fitted with gloves

lead to a considerable cost saving per year to Rheumatology and Therapy departments, especially as glove provision is a recurrent cost.

Whilst we did not test arthritis gloves in people with other forms of hand arthritis, it would be reasonable to assume similar results. Arthritis gloves are recommended by arthritis charities and medical information websites [37, 38] and commonly purchased by people with hand arthritis. Such recommendations should be reviewed. Health professionals should enable people with hand arthritis to consider carefully, informed by the evidence, whether to purchase gloves for themselves.

Even though the results demonstrated little benefit in hand symptoms and hand function from wearing either gloves, most people in both groups thought they were beneficial and would continue to wear them. Both groups thought gloves gave warmth, comfort and support [36]. Arthritis gloves are thought to impact on hand symptoms through applying pressure. The results suggest that pressure is not an active ingredient in arthritis gloves, as loose-fitting gloves led to similar results. Perceived benefits were more likely due to warmth. A previous trial concluded arthritis gloves and thermal gloves had similar effects [6]. We hypothesize that the tactile feedback from wearing gloves provided the reported feelings of comfort and support and may subconsciously have reminded users to take more care of their hand joints and subtly alter hand use during wear. A similar effect was also suggested in a pilot trial of thumb splints versus placebo thumb splints, which participants liked equally [39].

As both gloves gave similar results, and participants considered a main benefit was warmth [36], this suggests ordinary light-weight three-quarter finger gloves, made 
Table 3 Comparative effectiveness of intervention and placebo gloves at 12 weeks

\begin{tabular}{|c|c|c|c|c|c|c|c|c|}
\hline & \multicolumn{3}{|l|}{ Control } & \multicolumn{3}{|c|}{ Intervention } & \multirow[b]{2}{*}{$\begin{array}{l}\text { Adjusted between group } \\
\text { difference }{ }^{\mathrm{e}}(95 \% \mathrm{Cl})\end{array}$} & \multirow[b]{2}{*}{$\begin{array}{l}p \text { - } \\
\text { value }\end{array}$} \\
\hline & $\begin{array}{l}\text { Baseline } \\
\text { Mean } \\
\text { (S.D.) } \\
\text { Control } \\
n=103\end{array}$ & $\begin{array}{l}\text { 12-week follow- } \\
\text { up. Mean (S.D.) } \\
\text { Control } \\
n=79\end{array}$ & $\begin{array}{l}\text { Score } \\
\text { Change } \\
(0-12 w)\end{array}$ & $\begin{array}{l}\text { Baseline } \\
\text { Mean } \\
\text { (S.D.) } \\
n=103\end{array}$ & $\begin{array}{l}\text { 12-week follow- } \\
\text { up. Mean (S.D.) } \\
n=84\end{array}$ & $\begin{array}{l}\text { Score } \\
\text { Change } \\
(0-12 w)\end{array}$ & & \\
\hline $\begin{array}{l}\text { Dominant hand pain during } \\
\text { activity }\left(0-10 \text { NRS }{ }^{a}\right)\end{array}$ & $6.3(2.2)$ & $5.1(2.3)$ & 1.2 & $6.3(1.9)$ & $5.3(2.3)$ & 1.0 & $0.1(-0.47,0.67)$ & 0.72 \\
\hline $\begin{array}{l}\text { Non-dominant hand pain } \\
\text { during activity (0-10 NRS) }\end{array}$ & $5.6(2.4)$ & $4.5(2.4)$ & 1.1 & $5.7(2.4)$ & $4.3(2.5)$ & 1.4 & $-0.29(0.94,0.36)$ & 0.38 \\
\hline $\begin{array}{l}\text { Dominant hand pain during } \\
\text { rest (0-10 NRS) }\end{array}$ & $5.2(2.4)$ & $3.8(2.4)$ & 1.4 & $4.6(2.2)$ & $3.9(2.2)$ & 0.7 & $0.29(-0.32,0.90)$ & 0.35 \\
\hline $\begin{array}{l}\text { Non-dominant hand pain } \\
\text { during rest (0-10 NRS) }\end{array}$ & $4.7(2.7)$ & $3.6(2.4)$ & 1.1 & $4.4(2.5)$ & $3.3(2.3)$ & 1.1 & $-0.15(-0.81,0.51)$ & 0.66 \\
\hline $\begin{array}{l}\text { Dominant hand pain at } \\
\text { night } \\
\text { (0-10 NRS) }\end{array}$ & $5.5(2.8)$ & $3.8(2.6)$ & 1.4 & $5.0(2.5)$ & $4.0(2.5)$ & 1.4 & $0.34(-0.30,0.98)$ & 0.30 \\
\hline $\begin{array}{l}\text { Non-dominant hand pain at } \\
\text { night (0-10 NRS) }\end{array}$ & $4.9(2.9)$ & $3.5(2.7)$ & 1.4 & $4.6(2.8)$ & $3.2(2.5)$ & 1.4 & $-0.32(-0.98,0.35)$ & 0.35 \\
\hline $\begin{array}{l}\text { Hand stiffness in the } \\
\text { morning (Minutes) }\end{array}$ & $\begin{array}{l}141.1 \\
(272.8)\end{array}$ & $96.4(226.7)$ & 44.7 & $\begin{array}{l}132.7 \\
(269.8)\end{array}$ & $62.5(53.0)$ & 70.2 & $32(-0.25,15.83)$ & 0.37 \\
\hline $\begin{array}{l}\text { Hand stiffness dominant } \\
\text { hand (0-10 NRS) }\end{array}$ & $6.0(2.6)$ & $4.9(2.6)$ & 1.1 & $5.6(2.9)$ & $4.9(2.9)$ & 0.7 & $0.28(-0.41,0.97)$ & 0.42 \\
\hline $\begin{array}{l}\text { Hand stiffness non-dominant } \\
\text { hand (0-10 NRS) }\end{array}$ & $5.4(2.9)$ & $4.1(2.7)$ & 1.3 & $5.2(2.9)$ & $4.2(2.9)$ & 1.2 & $0.17(-0.55,0.89)$ & 0.64 \\
\hline $\begin{array}{l}\text { Self-reported dominant } \\
\text { hand condition (1-5) }\end{array}$ & $3.3(0.7)$ & $2.9(0.8)$ & 0.4 & $3.3(0.7)$ & $2.8(1.0)$ & 0.5 & $-0.18(-0.77,0.41)$ & 0.56 \\
\hline $\begin{array}{l}\text { Overall MAPHAND }{ }^{\mathrm{b}} \text { (Hand } \\
\text { function) (0-3) }\end{array}$ & $1.4(0.6)$ & $1.2(0.6)$ & 0.2 & $1.5(0.5)$ & $1.2(0.6)$ & 0.3 & $-0.05(-0.18,0.07)$ & 0.39 \\
\hline Overall HAQ ${ }^{c}(0-3)$ & $1.5(0.7)$ & $1.3(0.7)$ & 0.2 & $1.5(0.6)$ & $1.4(0.8)$ & 0.1 & $0(-0.13,0.13)$ & 0.99 \\
\hline Overall MHQ ${ }^{d}(0-100)$ & $\begin{array}{l}49.8 \\
(13.7)\end{array}$ & $57.2(17.1)$ & 7.4 & $\begin{array}{l}49.3 \\
(11.2)\end{array}$ & $57.0(17.0)$ & 7.9 & $0.37(-3.45,4.20)$ & 0.85 \\
\hline - Overall hand function & $\begin{array}{l}42.8 \\
(18.2)\end{array}$ & $50.3(22.8)$ & 7.5 & $\begin{array}{l}43.6 \\
(14.8)\end{array}$ & $54.6(19.9)$ & 11 & $3.49(-2.05,9.04)$ & 0.22 \\
\hline - Activities of daily living & $\begin{array}{l}51.1 . \\
(25.8)\end{array}$ & $59.7(27.4)$ & 8.6 & $\begin{array}{l}51.2 \\
(24.0)\end{array}$ & $59.0(27.1)$ & 7.8 & $-0.69(-6.10 ; 4.71)$ & 0.80 \\
\hline - Work & $\begin{array}{l}39.5 \\
(23.7)\end{array}$ & $50.9(23.1)$ & 11.4 & $\begin{array}{l}37.7 \\
(19.9)\end{array}$ & $48.5(26.0)$ & 10.8 & $-1.30(-7.28,4.69)$ & 0.67 \\
\hline - Pain & $\begin{array}{l}35.1 \\
(25.8)\end{array}$ & $33.6(23.4)$ & 1.5 & $\begin{array}{l}32.7 \\
(24.6)\end{array}$ & $33.1(23.4)$ & 0.4 & $-0.07(-6.54,6.40)$ & 0.98 \\
\hline - Aesthetics & $\begin{array}{l}65.5 \\
(19.5)\end{array}$ & $69.3(21.4)$ & 3.8 & $\begin{array}{l}64.2 \\
(18.5)\end{array}$ & $66.4(22.7)$ & 2.2 & $-2.18(-8.08,3.72)$ & 0.47 \\
\hline - Satisfaction & $\begin{array}{l}35.3 \\
(21.5)\end{array}$ & $48.5(26.6)$ & 13.2 & $\begin{array}{l}32.3 \\
(16.3)\end{array}$ & $48.4(25.4)$ & 16.4 & $1.95(-4.99,8.90)$ & 0.58 \\
\hline
\end{tabular}

${ }^{\mathrm{a}}$ NRS Numeric Rating Scale, ${ }^{\mathrm{b}}$ MAPHAND Measure of Activity Performance - Hand, ${ }^{c} H A Q$ Health Assessment Questionnaire, ${ }^{\mathrm{d}}$ MHQ Michigan Hand Questionnaire, eadjusted between group difference - adjusted for stratification variable (change in DMARDS) and baseline score

of nylon, cotton or wool (typically containing $5 \%$ elastane), purchased from High Street or online stores, could have similar effects. Therapists could recommend patients purchase such gloves instead. The time saved on glove provision could be used teaching hand exercises and joint protection, both of which are effective in reducing hand symptoms $[40,41]$. Future research could investigate whether people with arthritis consider wearing ordinary light-weight gloves affects hand status.
This is the first randomised controlled trial to evaluate arthritis glove-wear on daytime hand pain and function, which are the main reasons for arthritis gloves being prescribed currently in routine clinical practice. The results also confirmed previous smaller trials of full-finger arthritis gloves that indicated there are no differences in nocturnal pain and stiffness between wearing arthritis or placebo gloves [5]. This trial demonstrates the importance of testing widely used interventions, long 
Table 4 Mean (SD) health utility, Quality Adjusted Life Years (QALYS) and costs

\begin{tabular}{|c|c|c|c|c|c|c|}
\hline \multirow[b]{2}{*}{ EQ-5D-3L } & \multicolumn{3}{|l|}{ Control } & \multicolumn{3}{|l|}{ Intervention } \\
\hline & $\begin{array}{l}\text { Baseline } n= \\
103\end{array}$ & $\begin{array}{l}12 \text {-week follow-up } n= \\
79\end{array}$ & $\begin{array}{l}\text { Change } n= \\
79\end{array}$ & $\begin{array}{l}\text { Baseline } n= \\
103\end{array}$ & $\begin{array}{l}\text { 12-week follow-up } n= \\
84\end{array}$ & $\begin{array}{l}\text { Change } n= \\
84\end{array}$ \\
\hline Utility (Mean, SD) & $0.44(0.37)$ & $0.55(0.55)$ & $0.09(0.27)$ & $0.44(0.34)$ & $0.54(0.31)$ & $0.05(0.26)$ \\
\hline QALYs (Mean, SD) & $0.12(0.07)$ & & & $0.12(0.07)$ & & \\
\hline \multicolumn{7}{|c|}{ Costs during follow-up (Mean, SD) } \\
\hline Outpatient & \multicolumn{3}{|c|}{$£ 255(268)(n=78)$} & \multicolumn{3}{|c|}{$£ 317(333)(n=82)$} \\
\hline $\begin{array}{l}\text { Primary and community } \\
\text { care }\end{array}$ & \multicolumn{3}{|c|}{$£ 59(86)(n=75)$} & \multicolumn{3}{|c|}{$£ 66(101)(n=81)$} \\
\hline Intervention & \multicolumn{3}{|l|}{$£ 0$} & \multicolumn{3}{|l|}{$£ 129(n=78)$} \\
\hline TOTAL ${ }^{a}$ & \multicolumn{3}{|c|}{$£ 391(543)(n=75)$} & \multicolumn{3}{|c|}{$£ 552(464)(n=78)$} \\
\hline
\end{tabular}

${ }^{a}$ Total cost includes inpatient admissions, day case visits, and A\&E visits. The mean intervention cost is reported for participants with complete cost data. Intervention cost comprised of actual number of compression gloves provided per participant costing £31.43/glove (NHS dispensing costs) and one visit to a secondary care occupational therapist costing $£ 65.85$ (NHS reference costs)

established in clinical practice, for which there is little evidence. Identifying that arthritis gloves are not effective is a positive finding, as it enables clinical practice to be evidence-based. Guidelines for the provision of hand orthoses in arthritis should be updated. This trial also provides sound evidence on which patients can base their own decisions as to whether to purchase arthritis gloves or ordinary gloves instead, as the latter would be a considerable cost-saving, especially for those with limited incomes.

The strengths of our trial include the large sample size, longer follow-up than previous trials (12 weeks compared to 4 weeks) and full economic evaluation. Recruiting from 16 rheumatology out-patient departments increased the likelihood of a representative sample. The trial design was developed with the PPI group's and clinical therapists' inputs to ensure the control intervention was credible and procedures feasible. We ensured before the trial and during training that therapists understood about current evidence and the clinical equipoise between arthritis and placebo gloves, discussed any ethical concerns, and they were willing and able to credibly provide the placebo gloves. The self-reported glove adherence rates in both groups were similar indicating training supported therapists in placebo glove provision. We tested the make of arthritis glove known to provide the highest level of compression in order to maximise the ability to detect any positive effects on outcomes. It is therefore unlikely that any other make of arthritis glove providing less compression would result in different outcomes. The limitations of this trial are, like many non-pharmacological trials, that therapists could not be blinded. We did not have a third usual care only control group. We considered it important to offer treatment to all participants meeting trial criteria. An objective independent clinical hand assessment (e.g. of hand swelling and hand function) was not included. However, our feasibility study indicated the main changes were in pain, stiffness and daily hand function, which are reliably measurable through patient reported outcomes [14].

\section{Conclusion}

Arthritis gloves providing pressure and warmth and loose-fitting placebo gloves providing warmth, had only minimal effects on hand pain and function, with no differences between glove types. Participants perceived warmth as a main benefit from both gloves. Arthritis gloves were not cost-effective. Given these results, therapists could recommend patients buy ordinary threequarter finger length gloves. These are widely available, would save health services money and be low-cost for patients. These findings inform evidence-based treatment choices for clinicians in specialist musculoskeletal services, and community services, and for patients considering purchasing arthritis gloves.

Table 5 Results of incremental cost-effectiveness analysis for intervention versus control gloves

\begin{tabular}{|c|c|c|c|c|c|c|}
\hline & \multirow{2}{*}{$\begin{array}{l}\text { Net cost } \\
(95 \% \mathrm{Cl})\end{array}$} & \multirow{2}{*}{$\begin{array}{l}\text { Net QALY } \\
(95 \% \mathrm{CI})\end{array}$} & \multirow{2}{*}{$\begin{array}{l}\text { ICER } \\
\text { (£/QALY) }\end{array}$} & \multicolumn{3}{|c|}{ Probability intervention is cost effective versus control if WTPT = } \\
\hline & & & & $£ 20,000 /$ QALY & $£ 30,000 / Q A L Y$ & $£ 60,000 /$ QALY \\
\hline Complete cases (ITT) $(n=151)$ & $\begin{array}{l}£ 251 \\
(106,396)\end{array}$ & $\begin{array}{c}0.003 \\
(-0.017,0.023)\end{array}$ & $£ 83,700 / Q A L Y$ & 0.19 & 0.29 & 0.44 \\
\hline
\end{tabular}

Note: whole $£$ reported in table but ICERs calculated including pence

Covariates costs: pre-baseline costs, stratification variable

Covariates QALYs: baseline health status, stratification variable

ICER incremental cost-effectiveness ratio, WTPT willingness to pay threshold, ITT intention to treat

a at different willingness to pay thresholds, based on 10,000 bootstrap simulations 


\section{Supplementary Information}

The online version contains supplementary material available at https://doi. org/10.1186/s12891-020-03917-8.

Additional file 1: Tables S1 to S5. A-Gloves 12-week follow-up questionnaire

\section{Abbreviations}

A\&E: Accident and Emergency; A-GLOVES: Arthritis Gloves trial; $\mathrm{Cl}$ : Confidence interval; DMARDs: Disease modifying anti-rheumatic drugs; HAQ: Health Assessment Questionnaire; ICER: Incremental cost-effectiveness ratio; IQR: Inter-quartile range; ITT: Intention to treat; MCPJs: Metacarpophalangeal joints; MAPHAND: Measure of Activity Performance Hand; MHQ: Michigan Hand Outcomes Questionnaire; MCID : Minimal clinically important difference; MDC: Minimal Detectable Change; NHS: National Health Service; NRS : Numeric rating scale; PPI : Patient and public involvement; PIPJs: Proximal interphalangeal joints; QALY: Quality Adjusted Life Year; RA: Rheumatoid arthritis; SD: Standard deviation; UIA: Undifferentiated inflammatory arthritis; UK: United Kingdom; WTPT: Willingness to pay threshold

\section{Acknowledgements}

This trial was undertaken with the support of Lancashire Clinical Trials Unit (CTU), University of Central Lancashire, Preston, UK. The authors would like to thank Denise Forshaw, Principal Clinical Trial Manager, and all staff in the CTU involved in the study, Glenn Holt, Alison Hedley and Jane Fitzgerald. We would also like to thank: all the trial participants for their time and support; Wendy Jones and Barbara Birtwistle (patient research partners) for their invaluable advice during trial planning, focus group participation and throughout; Dr. Helen Myers, Centre for Primary Care Research, Keele University, focus group participation; Angela Jacklin, Rheumatology occupational therapist, Mid Cheshire Hospitals NHS Foundation Trust, focus group participation and assisting with training therapists in glove provision; Nazina Arafin (trial assistant, Centre for Health Services Research, University of Salford); Owen Summers (Research Contracts, University of Salford); the R\&D department and Finance Office, Salford Royal NHS Foundation Trust for assistance with grant financial planning and management; members of the Trial Steering Committee (Chair: Dr. Peter Klimiuk, Consultant Rheumatologist, Pennine MSK Partnership, Oldham; Mike Bradburn, Senior Medical Statistician, Sheffield Clinical Trials Unit, SCHARR, University of Sheffield; Cathy Ball, Research Clinical Specialist Hand Therapist, Kennedy Institute for Rheumatology, Oxford; Dr. Michael Callaghan, Research Fellow, University of Manchester); and the participating clinicians and sites for their support: Prof David Walsh, Debbie Wilson, Rachael Murphy, Charlotte Brooks, Phil Avery (Sherwood Forest Hospitals NHS Foundation Trust); Nicky Walker, Jan Colclough, Helen Sawle (Mid Cheshire Hospitals NHS Foundation Trust); Yvonne Hough and Denise Graham (St Helens and Knowsley Teaching Hospitals NHS Trust); Dr. Jill Firth, Charlotte Critchley, Helen Light, Katherine Kinsey (Pennine MSK Partnership, Oldham); Mary Dooley (Southport and Ormskirk Hospital NHS Trust): Anne Boulton (Northumbria Healthcare NHS Foundation Trust); Jo Harness, Jane Hunt (Northern Devon Healthcare NHS Trust); Janet Perkins (Manchester University NHS Foundation Trust); Dervil Dockrell, Sarah Wilson (Sheffield Teaching Hospitals NHS Foundation Trust); Jill Spicer, Christine Mate (The Royal Wolverhampton NHS Trust); Anne Bontoft, Sandra Lyle, Carol Gray (Northern Lincolnshire and Goole NHS Foundation Trust); Aviva Richards (Pennine Acute Hospitals NHS Trust); Carole Graham (Midlands Partnership NHS Foundation Trust); Christine Beck, Kirstie Neat (Leeds Teaching Hospitals NHS Trust); Nicky Gray (NHS Borders); Kate McAlarey, Patricia Bissett (NHS Greater Glasgow and Clyde).

\section{Authors' contributions}

All authors contributed to study design, interpretation of data; critical revision of the report; approved the final manuscript of the report; and agree to be accountable for all aspects of the work. AH, YP, SC, CS, EC, WJ, JA, YH, JF and TO'N conceived the study. AH and YP contributed to acquisition of baseline data. SC and $\mathrm{CH}$ analysed the clinical outcomes, and EC completed the health economic analysis. AH drafted the first report, with input from SC, CS and EC. AH was the Chief Investigator.

\section{Funding}

This paper presents independent research funded by the National Institute of Health Research (NIHR) under its Research for Patient Benefit (RfPB) Programme (Grant Reference Number PB-PG-0214-33010). The views expressed are those of the authors and not necessarily those of the NHS, the NIHR or the Department of Health and Social Care.

\section{Availability of data and materials}

The datasets used and/or analysed during the current study are available from the corresponding author on reasonable request. Clinical and trial procedures are described in the A-GLOVES: Occupational Therapy Glove Provision manual v2, freely available at http://usir.salford.ac.uk/42272

\section{Ethics approval and consent to participate}

The trial was approved by the North of Scotland National Research Ethics Service Committee (REC reference 15/NS/0077) and the University of Salford School of Health Sciences Research, Innovation and Academic Engagement Ethical Approval Panel (HSCR 15-94). Patients gave written informed consent in accordance with the principles of Good Clinical Practice and the Declaration of Helsinki. Photographs are of the first author's hand.

\section{Consent for publication}

Not applicable.

\section{Competing interests}

The authors have no financial relationship relevant to this article to disclose. The authors have no conflicts of interest to disclose.

\section{Author details}

${ }^{1}$ Centre for Health Sciences Research, School of Health and Society, University of Salford, Allerton Building, Frederick Road, Salford M6 6PU, UK. ${ }^{2}$ Centre for Biostatistics, Division of Population Health, Health Services Research and Primary Care, University of Manchester, Manchester, UK ${ }^{3}$ Manchester Centre for Health Economics, Division of Population Health, Health Services Research and Primary Care, University of Manchester, Manchester, UK. ${ }^{4}$ Health Sciences, University of Southampton, Southampton, UK. ${ }^{5}$ Rheumatology Occupational Therapy, St Helens and Knowsley Teaching Hospitals NHS Trust, St Helens, UK. ${ }^{6}$ Centre for Epidemiology Versus Arthritis, Division of Musculoskeletal and Dermatological Sciences, Manchester Academic Health Science Centre, University of Manchester, Manchester, UK ${ }^{7} \mathrm{NIHR}$ Manchester Biomedical Research Centre, Manchester University NHS Foundation Trust, Manchester, UK. ${ }^{8}$ Pennine MSK Partnership, Oldham, Manchester, UK.

Received: 14 July 2020 Accepted: 23 December 2020

Published online: 08 January 2021

\section{References}

1. World Health Organisation. Chronic rheumatic conditions. Available from https://www.who.int/chp/topics/rheumatic/en/. Cited 2019 Nov 27.

2. Seto $Y$, Inoue E, Shikara K, Hoshi D, Sugimoto N, Sato E, et al. Functional disability can deteriorate despite suppression of disease activity in patients with rheumatoid arthritis: a large observational cohort study. Med Rheumatol. 2013;23:1179-85.

3. Horsten NCA, Ursum J, Roorda LD, van Schaardenburg D, Dekker J, Hoeksma AF. Prevalence of hand symptoms, impairments and activity limitations in rheumatoid arthritis in relation to disease duration. J Rehabil Med. 2010;42:916-21.

4. Prior $Y$, Hammond $A$. The survey of rheumatology occupational therapy glove practice and provision in the UK. Rheumatology. 2015;54:1101.

5. Hammond A, Jones $V$, Prior $Y$. The effects of compression gloves on hand symptoms and hand function in rheumatoid arthritis and hand osteoarthritis: a systematic review. Clin Rehabil. 2016;30(3):213-24.

6. Oosterveld FJG, Rasker JJ. The effect of pressure gradient and thermolactyl control gloves in arthritis patients with swollen hands. Br J Rheumatol. 1990; 29:197-200.

7. McKnight PT, Kwoh CK. Randomised controlled trial of pressure gloves in rheumatoid arthritis. Arthritis Care Res. 1999:5:223-7.

8. Isotoner Gloves information from Isotoner ${ }^{\oplus}$ [Internet]. Available from https:// www.isotoner.com/p/isotoner-fingerless-therapeutic-gloves-25830. Cited 2020 Feb 3. 
9. Jobskin classic oedema gloves information from Jobskin ${ }^{\oplus}$. Available from https://www.jobskin.co.uk/classic-oedema-glove. Updated 2020 Jan 13; Cited 2020 Feb 3.

10. Research Nester. Therapy Gloves Market Segmentation by Size (Small, Medium, Large); By Product Type (Compression gloves, thermal gloves and splint gloves); By Design (Open Finger Gloves, Full Finger Gloves and Wrist Wraps); By Material Type (Cotton and Synthetic Fibre); By End User (Physiotherapy Centres; Home Care and Others); - Global Demand Analysis and Opportunity Outlook 2020-2027. Available from: https://www. researchnester.com/reports/therapy-gloves-market/1882. Cited 2020 May 23.

11. Culic DD, Battaglia MC, Wichman C, Schmid FR. Efficacy of compression gloves in rheumatoid arthritis. Am J Phys Med. 1979;58:278-84.

12. Swezey RL, Spiegel TM, Cretin S, Clements P. Arthritic hand response to pressure gradient gloves. Arch Phys Med Rehabil. 1979;60:375-7.

13. Dixon A, St J, Harrison RA, Pocock P. Trial of Isotoner gloves for morning stiffness and pain in rheumatoid arthritis. Br J Clin Pract. 1986;40:271-2.

14. Hammond A, Prior $Y$, Jones $V$, Dooley $M$, Hough $Y$, Jacklin A. A pre-test post-test pilot trial of compression gloves in early inflammatory and rheumatoid arthritis. Rheumatology. 2015;54:1111-2. https://doi.org/10.1093/ rheumatology/kev089.045.

15. Prior Y, Sutton C, Cotterill S, Adams J, Camacho E, Arafin N, et al. The effects of arthritis gloves on people with rheumatoid arthritis: or inflammatory arthritis with hand pain: a study protocol for a multicentre randomised controlled trial (the A-GLOVES trial). BMC Musculoskeletal Dis. 2017;18:224.

16. Kiltz U, von Zabern U, Baraliakos X, Heldman F, Mintrop B, Sarholz M, et al. Diagnostic value of a 3-day course of prednisolone in patients with possible rheumatoid arthritis - the TryCort study. Arth Res Ther. 2017;19:273.

17. Sealed Envelope. Available from https://www.sealedenvelope.com/. Cited 2020 Feb 18.

18. Looking After Your Joints when you have arthritis from VersusArthritis.org. Available from https://www.versusarthritis.org/media/1271/looking-afteryour-joints-information-booklet.pdf. Published 2013 June; Cited 2020 April 21.

19. Heine PJ, Williams MA, Williamson E, Bridle C, Adams J, O'Brien A, et al. Development and delivery of an exercise intervention for rheumatoid arthritis: strengthening and stretching for rheumatoid arthritis of the hand (SARAH) trial. Physiotherapy. 2012;98:121-30.

20. Hammond A, Jacklin A, Hough Y. A-GLOVES: Occupational Therapy Glove Provision Manual version 2: University of Salford; 2016. http://usir.salford.ac. uk/42272 Accessed 18 Feb 2020.

21. Jones LE, White P, Donovan-Hall M, Hislop K, Boucas SB, Adams J. The thoughts and feelings held by clinicians about the delivery of a placebo thumb splint in an osteoarthritis rehabilitation trial. Hand Therapy. 2013;18:77-83.

22. Prior Y, Hammond A, Arafin N, Adams J, Hough Y, Jacklin A. Rheumatology occupational therapist' views of participating in a randomised controlled trial of arthritis gloves. Rheumatology. 2017;56(Suppl. 2):kex062.121. https:// doi.org/10.1093/rheumatology/kex062.121

23. Hawker GA, Mian S, Kendzerska T, French M. Measures of adult pain Arthritis Care Res. 2011;63(Suppl 11):S240-52.

24. Wolfe F, Wolfe F, Michaud K. Assessment of pain in rheumatoid arthritis: minimal clinically significant difference, predictors, and the effect of antitumor necrosis factor therapy. J Rheumatol. 2007;34:1674-8.

25. Strand V, Boers M, Idzerda L, Kirwan JR, Kvien TR, Tugwell PS, et al. It's good to feel better but It's better to feel good and even better to feel good as soon as possible for as long as possible. Response criteria and the importance of change at OMERACT 10. J Rheumatol. 2011;38:1720-7.

26. Strand V, Schiff M, Tundia M, Friedman A, Meerwein S, Pangan A, et al. Effects of upadacitinib on patient-reported outcomes: results from SELECTBEYOND, a phase 3 randomized trial in patients with rheumatoid arthritis and inadequate responses to biologic disease-modifying antirheumatic drugs. Arthritis Res Ther. 2019;21:263.

27. Prior Y, Tennant A, Tyson S, Kjeken I, Hammond A. Measure of activity performance of the hand (MAP-hand) questionnaire: linguistic validation cultural adaptation and psychometric testing in people with rheumatoid arthritis in the UK. BMC Musculoskelet Disord. 2018;19:275.

28. Chung KC, Pillsbury MS, Walters MR, Hayward RA. Reliability and validity testing of the Michigan hand outcomes questionnaire. J Hand Surg [Am]. 1998;23:575-87.

29. London D, Stepan JG, Calfee RP. Determining the Michigan hand outcomes questionnaire minimal clinically important difference by means of three methods. Plast Reconstr Surg. 2014;133(3):616-25.
30. Kirwan JR, Reeback JS. Stanford health assessment questionnaire modified to assess disability in British patients with rheumatoid arthritis. $\mathrm{Br} \mathrm{J}$ Rheumatol. 1986;25:206-9.

31. EuroQol group. EuroQol - a new facility for the measurement of healthrelated quality of life. Health Policy. 1990;16:199-208.

32. StataCorp. Stata Statistical Software: Release 14. College Station: StataCorp LP; 2015.

33. Personal Social Services Research Unit, Unit Costs of Health and Social Care 2016. University of Kent. Available from https://www.pssru.ac.uk/pub/uc/uc2 016/full.pdf. Cited 2020 April 24.

34. NHS Improvement. NHS Reference Costs 2016-17. Available from https:// improvement.nhs.uk/documents/741/Reference_costs_collection_ guidance_201617.pdf. Published 2017 Feb; cited 2020 April 29.

35. Dolan P, Gudex C, Kind P, Williams A. The time trade-off method: results from a general population study. Health Econ. 1996;5(2):141-54

36. Prior Y, Arafin N, Bartley C, Hammond A. Inflammatory or rheumatoid arthritis patients' perspectives on the effect of arthritis gloves on their hand pain and function (A-GLOVES TRIAL): a qualitative study. Ann Rheum Dis. 2018. https://doi.org/10.1136/annrheumdis-2018-eular.5980.

37. IMAK $^{\circledast}$ Arthritis gloves information. Arthritis Foundation ${ }^{\oplus}$. Available from https://www.arthritis.org/living-with-arthritis/tools-resources/ease-of-use/ health-and-wellness/imak-arthritis-gloves.php. Cited 2020 Feb 18.

38. Rheumatoid Arthritis gloves information. WebMD ${ }^{\star}$, Available from https://www webmd.com/rheumatoid-arthritis/ra-gloves. Updated 2018; cited 2020 Feb 18.

39. Adams J, Barbosa Bouças S, Hislop K, Dziedzic K, Arden N, McKenna C, et al. The effectiveness and efficacy of splints for Thumb Base osteoarthritis: a pilot randomized controlled trial. Rheumatology. 2014;53(Suppl):i41-2. https://doi.org/10.1093/rheumatology/keu090.005.

40. Lamb SE, Williamson EM, Heine PJ, Adams J, Dosanjh S, Dritsaki M, et al. Exercises to improve function of the rheumatoid hand (SARAH): a randomised controlled trial. Lancet. 2015;385(9966):421-9.

41. Hammond A, Freeman K. The long-term outcomes from a randomised controlled trial of an educational-behavioural joint protection programme for people with rheumatoid arthritis. Clin Rehabil. 2004;18:520-8.

\section{Publisher's Note}

Springer Nature remains neutral with regard to jurisdictional claims in published maps and institutional affiliations.
Ready to submit your research? Choose BMC and benefit from:

- fast, convenient online submission

- thorough peer review by experienced researchers in your field

- rapid publication on acceptance

- support for research data, including large and complex data types

- gold Open Access which fosters wider collaboration and increased citations

- maximum visibility for your research: over $100 \mathrm{M}$ website views per year

At $\mathrm{BMC}$, research is always in progress.

Learn more biomedcentral.com/submissions 